\title{
Analysis of Influence of the Inner Diameter of the Permanent Magnet on Rotor Stress and Critical Speed for the High Speed Permanent Magnet Motor
}

\author{
Xu Zhiyu, Deng Zhiquan, Zhang Zhongming, Sun Quangui, Pang Gucai \\ Department of Electrical Engineering, Nanjing University of Aeronautics and Astronautics, Nanjing, China
}

\section{Email address:}

952495152@qq.com (Xu Zhiyu),dzq@nuaa.edu.cn (Deng Zhiquan)

\section{To cite this article:}

Xu Zhiyu, Deng Zhiquan, Zhang Zhongming, Sun Quangui, Pang Gucai. Analysis of Influence of the Inner Diameter of the Permanent Magnet on Rotor Stress and Critical Speed for the High Speed Permanent Magnet Motor. Science Discovery. Vol. 6, No. 5, 2018 , pp. 406-413. doi: 10.11648/j.sd.20180605.25

Received: September 30, 2018; Accepted: October 15, 2018; Published: October 18, 2018

\begin{abstract}
While the rotor of a high-speed permanent magnet motor runs at high speed, it is difficult for the permanent magnet to bear the huge tensile stress which caused by centrifugal force. In addition, the rotor is also prone to vibration and even bending deformation due to resonance. Therefore, the strength and critical speed of the rotor must be analyzed and checked. In this paper, because of the slim characteristic of high speed permanent magnet rotors, the displacement and stress fields of a $150 \mathrm{~kW}, 30000$ $\mathrm{r} /$ min surface mounted high speed permanent magnet motor rotor are deduced based on the theory of the thick-walled cylinder. The finite element method is used to verify the correctness of the analytical calculation. Based on the deduced analytical formulas, the influences of the inner diameter of the permanent magnet and the sleeve material on the rotor stress are analyzed. At the same time, the finite element method is used to calculate the critical speed of the rotor and analysis the influences of the inner diameter of the permanent magnet and the sleeve material on the critical speed of the rotor shaft system. A basis for the design of the high speed permanent magnet motor rotor is given.
\end{abstract}

Keywords: High Speed Permanent Magnet Motor, Inner Diameter of Permanent Magnet, Stress, Critical Speed

\section{永磁体内径对高速永磁电机转子应力和临界转速的影响分析}

许治宇, 邓智泉, 张忠明, 孙权贵, 庞古才

电气工程系, 南京航空航天大学, 南京, 中国

邮箱

952495152@qq.com（许治宇）, dzq@nuaa.edu.cn (邓智泉)

摘要: 高速永磁电机转子在高速运行时, 永磁体难以承受由离心力引起的巨大拉应力, 转子也容易因共振产生较大的振 动甚至弯曲变形, 因此必须对转子强度和临界转速进行分析校核。本文针对高速转子细长型结构的特点, 基于厚壁圆筒 理论, 对一台 $150 \mathrm{~kW}, 30000 \mathrm{r} / \mathrm{min}$ 的表贴式高速永磁电机转子的位移场和应力场进行了解析计算, 采用有限元法验证了 解析计算的正确性, 基于推导的解析公式, 分析了永磁体内径、护套材料对转子应力的影响; 同时采用有限元法对转子 临界转速进行计算, 分析了永磁体内径、护套材料对转子轴系临界转速的影响; 为高速永磁电机转子的设计提供依据。

关键词: 高速永磁电机, 永磁体内径, 应力, 临界转速 


\section{1. 引言}

高速永磁电机具有转速高、体积小、功率密度高和效 率高等特点, 在诸多领域(如高速机床、压缩机、鼓风机、 储能飞轮等)都有着广泛的应用前景, 目前已经成为电机 研究领域的一个热点[1-5]。

高速永磁电机转子的最高转速受转子结构、尺寸和材 料的影响 [6]。目前, 高速永磁电机转子中的永磁体大多 采用钐钴或者钕铁嗍永磁材料, 其抗拉强度较低, 在高转 速下容易被离心力破坏, 现在普遍采用的方法是在永磁体 表面过盈装配护套对永磁体进行保护 $[7,8]$ 。此外, 转子在 高速旋转过程中, 转子振动是制约电机正常运行的瓶颈, 为了避免出现转子共振、弯曲的情况, 必须对转子进行动 力学分析[9-11], 准确计算转子系统的临界转速。

目前高速永磁电机最为常用的转子结构是表贴式结 构(surface mounted permanent, SPM)[12,13]。国内外学者 对表贴式结构的高速永磁电机转子应力和动力学问题做 了相关研究。文献[6]对高速永磁电机的设计和分析进行了 系统的概述, 阐述了对转子应力和动力学研究的必要性。 文献[14]对高速永磁电机采用两种转子结构时的应力情况 进行了对比, 对转子结构强度进行了优化设计。文献[15] 对高速永磁电机采用合金护套和碳纤维复合材料护套时 的应力问题进行了比较研究, 总结了两种保护措施的设计 规律。文献[16]分析了表贴式高速永磁电机转子中静态过 盈量、转轴材料特性、护套厚度对转子强度的影响。文献 [17]对采用碳纤维护套的高速永磁电机转子进行了转子动 力学分析和临界转速计算。文献 $[18,19]$ 对磁轴承——转子 系统进行了动力学分析和临界转速计算。文献[20]对用于 计算转子临界转速的限元法中的法向刚度因子进行了优 化。

目前已发表的文献对于表贴式结构的高速永磁电机 转子的研究, 都仅仅是基于已经设计确定的永磁体尺寸对 转子的应力或者动力学作分析, 没有研究永磁体尺寸变化 对转子应力和动力学的影响。此外, 对于现在常用的两种 非导磁合金护套：钛合金护套和镍基合金护套，也没有相 关文献资料分析比较它们对转子强度和临界转速的影响。

本文针对一台设计参数为 $150 \mathrm{~kW}, 30000 \mathrm{r} / \mathrm{min}$ 的高速 永磁电机, 改变永磁体内径, 调整相应的永磁体外径保证 电机的电磁性能相同, 对具有不同尺寸永磁体的转子进行 应力和动力学分析, 研究永磁体内径对转子机械性能的影 响; 同时分别采用钛合金护套和镍基合金护套，分析这两 种护套对转子应力和临界转速的影响, 为高速永磁电机转 子设计提供依据。

\section{2. 电磁设计}

本文对一台设计参数为 $150 \mathrm{KW}, 30000 \mathrm{r} / \mathrm{min}$ 的高速 永磁电机进行电磁方案设计, 电机转子采用表贴式结构, 如图1所示。其中护套分别采用钛合金和镍基合金材料, 永磁体采用钕铁嗍材料, 转轴采用 $40 \mathrm{CrNiMoA}$ 材料。电机 的设计参数如表 1 所示。

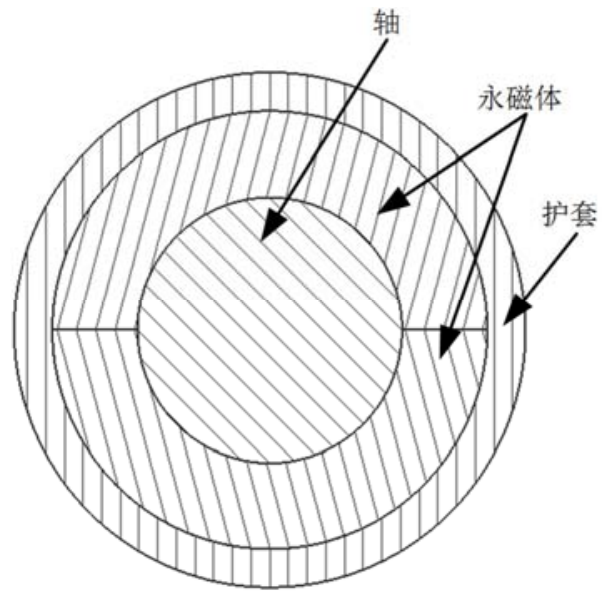

图1 转子结构。

表1 电机参数。

\begin{tabular}{ll}
\hline 参数 & 参数值 \\
\hline 额定功率 $/ \mathrm{KW}$ & 150 \\
额定转速 $/ \mathrm{rpm}$ & 30000 \\
额定电压 $/ \mathrm{V}$ & 380 \\
额定频率 $/ \mathrm{Hz}$ & 500 \\
极数 & 2 \\
铁心长度 $/ \mathrm{mm}$ & 122.8 \\
定子内径 $/ \mathrm{mm}$ & 92 \\
\hline
\end{tabular}

保持电机的电磁性能相同, 分析永磁体内径、护套材 料对转子应力和临界转速的影响。这里作如下设定: 保持 电机的定子结构不变, 仅改变转子中的相关尺寸。转子尺 寸设计如下: 改变永磁体内径, 以获得相同的气隙磁密为 目标, 通过有限元仿真确定相应的永磁体外径, 从而得到 一系列永磁体尺寸, 如表2所示。可以看出随着永磁体内 径的增加, 为保持气隙磁密不变, 永磁体外径也在持续增 加, 但永磁体的厚度在减小。

表2 永磁体尺寸和气隙磁密。

\begin{tabular}{lll}
\hline 永磁体内径 $/ \mathbf{m m}$ 永磁体外径/ $\mathbf{m m}$ 气隙磁密/T \\
\hline 30 & 70.25 & 0.5736 \\
35 & 71.5 & 0.577 \\
40 & 72.75 & 0.5774 \\
45 & 74 & 0.5745 \\
50 & 75.5 & 0.5744 \\
55 & 77.25 & 0.5782 \\
60 & 79 & 0.5702 \\
\hline
\end{tabular}

\section{3. 转子强度分析}

永磁体在高速旋转下会承受较大的拉应力, 必须对转 子进行强度分析。本文首先采用理论解析法推导了表贴式 高速永磁电机转子的应力场、位移场和应变场的解析模型, 之后再通过有限元法验证了解析模型的准确性。基于推导 的应力解析模型分析永磁体内径对于转子应力的影响, 同 时比较钛合金护套和镍基合金护套对永磁体的保护效果。

\section{1. 符号定义}

为方便理论公式的推导, 进行如下的符号定义, 包括 各物理量和下标, 如表3所示。 
表3 符号定义。

\begin{tabular}{ll}
\hline 符号 & 定义 \\
\hline $\mathrm{u}$ & 位移量 \\
$\sigma$ & 应力 \\
$\mathrm{r}$ & 半径 \\
$\omega$ & 转速 \\
$\mu$ & 泊松比 \\
$\mathrm{E}$ & 弹性模量 \\
$\rho$ & 密度 \\
$\delta$ & 过盈量 \\
下标 $z$ & 转轴参数 \\
下标 $m$ & 永磁体参数 \\
下标 $s$ & 护套参数 \\
下标 $r$ & 径向参数 \\
下标 $\theta$ & 切向参数 \\
下标 $i$ & 内径处参数 \\
下标 $o$ & 外径处参数 \\
\hline
\end{tabular}

\section{2. 表贴式高速永磁电机转子强度分析}

采用弹性力学中的平面应力模型对转子进行强度分 析。根据转轴、永磁体、合金护套三者间的配合关系和边 界条件, 可以求解出各转子部件间的接触面上的接触压强。 再根据这些接触压强, 求解出转子各部件中对应的应力和 位移。

其中转子的平衡方程为:

$$
\sigma_{\theta}-\sigma_{r}-r \frac{d \sigma_{r}}{d r}=\rho \omega^{2} r^{2}
$$

转子微元体的几何方程为:

$$
\left\{\begin{array}{l}
\varepsilon_{r}=\frac{d u}{d r} \\
\varepsilon_{\theta}=\frac{u}{r}
\end{array}\right.
$$

对于平面应力模型, 由胡克定律可得本构方程:

$$
\left\{\begin{array}{l}
\varepsilon_{r}=\frac{1}{E}\left(\sigma_{r}-\mu \sigma_{\theta}\right)+\alpha \Delta T \\
\varepsilon_{\theta}=\frac{1}{E}\left(\sigma_{\theta}-\mu \sigma_{r}\right)+\alpha \Delta T
\end{array}\right.
$$

联立(2)、(3)两式, 可得转子中径向力和切向力与转 子位移的关系:

$$
\left\{\begin{array}{l}
\sigma_{r}=\frac{E}{1-\mu^{2}}\left[\frac{d u}{d r}+\mu \frac{u}{r}-(1+\mu) \alpha \Delta T\right] \\
\sigma_{\theta}=\frac{E}{1-\mu^{2}}\left[\mu \frac{d u}{d r}+\frac{u}{r}-(1+\mu) \alpha \Delta T\right]
\end{array}\right.
$$

\subsection{1. 合金护套的应力和位移分析}

如图1所示, 护套在高速旋转中, 假设内表面受均匀 压强p 2 的作用; 护套外表面所受外力为 0 , 即护套内壁的 径向正应力为 $-\mathrm{p} 2$, 外表面的径向正应力为 0 。结合转子微 元体的平衡方程、几何方程和本构方程, 考虑护套内外表 面的应力边界条件，可得到护套的径向位移，径向应力和 切向应力。径向位移为:

$$
u_{s r}=C_{1 s} r+\frac{C_{2 s}}{r}-\frac{\rho_{s} \omega^{2} r^{3}\left(1-\mu_{s}^{2}\right)}{8 E_{s}}+\frac{\alpha_{s}\left(1+\mu_{s}\right)}{r} \int_{R_{S i}}^{r} \Delta T r d r
$$

其中, $C_{I s}$ 和 $C_{2 s}$ 为微分常数, 与护套尺寸、材料属性和 边界条件相关。

在离心力和均匀压强 $\mathrm{p} 2$ 的作用下，护套的径向应力 $\sigma_{s r}$ 和切向应力 $\sigma_{s \theta}$ 分别为:

$$
\begin{aligned}
& \sigma_{s r}=\frac{E_{S}}{1-\mu_{S}^{2}}\left[C_{1 s}\left(1+\mu_{s}\right)+\frac{C_{2 s}\left(\mu_{s}-1\right)}{r^{2}}\right]-\frac{\rho_{s} \omega^{2} r^{2}\left(3+\mu_{s}\right)}{8}-\frac{\alpha_{s} E_{S}}{r^{2}} \int_{R_{S i}}^{r} \Delta T r d r \\
& \sigma_{S r}=\frac{E_{S}}{1-\mu_{s}^{2}}\left[C_{1 s}\left(1+\mu_{s}\right)+\frac{C_{2 s}\left(1-\mu_{s}\right)}{r^{2}}\right]-\frac{\rho_{S} \omega^{2} r^{2}\left(1+3 \mu_{s}\right)}{8}+\frac{\alpha_{S} E_{S}}{r^{2}} \int_{R_{S i}}^{r} \Delta T r d r
\end{aligned}
$$

\subsection{2. 永磁体的应力和位移分析}

如图1所示, 该转子中, 永磁体外表面通过过盈配合装配护套, 设过盈量为 $\delta$, 永磁体内表面与转轴外表面之间 为零过盈配合。假设永磁体内表面受接触压强p1作用，永磁体外表面受接触压强p2作用。

类似的, 根据相关方程和力学定理, 考虑相应的边界条件, 可得永磁体在上述力作用下的径向位移, 径向应力和 切向应力。径向位移为:

$$
u_{m r}=C_{1 m} r+\frac{C_{2 m}}{r}-\frac{\rho_{m} \omega^{2} r^{3}\left(1-\mu_{m}^{2}\right)}{8 E_{S}}+\frac{\alpha_{m}\left(1+\mu_{m}\right)}{r} \int_{R_{m i}}^{r} \Delta T r d r
$$

永磁体的径向应力和切向应力分别为:

$$
\begin{aligned}
& \sigma_{m r}=\frac{E_{m}}{1-\mu_{m}^{2}}\left[C_{1 m}\left(1+\mu_{m}\right)+\frac{C_{2 m}\left(\mu_{m}-1\right)}{r^{2}}\right]-\frac{\rho_{m} \omega^{2} r^{2}\left(3+\mu_{m}\right)}{8}-\frac{\alpha_{m} E_{m}}{r^{2}} \int_{R_{m i}}^{r} \Delta T r d r \\
& \sigma_{s r}=\frac{E_{m}}{1-\mu_{m}^{2}}\left[C_{1 m}\left(1+\mu_{m}\right)+\frac{C_{2 m}\left(1-\mu_{m}\right)}{r^{2}}\right]-\frac{\rho_{m} \omega^{2} r^{2}\left(1+3 \mu_{m}\right)}{8}+\frac{\alpha_{m} E_{m}}{r^{2}} \int_{R_{m i}}^{r} \Delta T r d r
\end{aligned}
$$

其中, $C_{I m}$ 和 $C_{2 m}$ 为微分常数, 与护套尺寸、材料属性和边界条件相关。

\subsection{3. 转轴的应力和位移分析}

如图1所示, 转轴外表面与永磁体接触, 其接触压强为 $\mathrm{p} 1$, 且在轴心处径向位移为 0 , 同理, 可得转轴的径向位移 为: 


$$
u_{z r}=C_{1 z} r+\frac{C_{2 z}}{r}-\frac{\rho_{z} \omega^{2} r^{3}\left(1-\mu_{z}^{2}\right)}{8 E_{z}}+\frac{\alpha_{z}\left(1+\mu_{z}\right)}{r} \int_{R_{z i}}^{r} \Delta \operatorname{Tr} d r
$$

其中, $C_{1 r} 、 C_{2 r}$ 为微分常数, 由转轴的尺寸、材料属性和边界条件确定, 由于在轴心处径向位移为 0 , 易得 $C_{2 r}$ 为 0 。 转轴的径向应力和切向应力分别为:

$$
\begin{aligned}
& \sigma_{z r}=\frac{E_{z}}{1-\mu_{z}^{2}} \times C_{1 z}\left(1+\mu_{z}\right)-\frac{\rho_{z} \omega^{2} r^{2}\left(3+\mu_{z}\right)}{8}-\frac{\alpha_{z} E_{z}}{r^{2}} \int_{R_{z i}}^{r} \Delta T r d r \\
& \sigma_{z \theta}=\frac{E_{z}}{1-\mu_{z}^{2}} \times C_{1 z}\left(1+\mu_{z}\right)-\frac{\rho_{z} \omega^{2} r^{2}\left(1+3 \mu_{z}\right)}{8}+\frac{\alpha_{z} E_{z}}{r^{2}} \int_{R_{z i}}^{r} \Delta T r d r
\end{aligned}
$$

\subsection{4. 接触压强 $\mathrm{p} 1$ 和 $\mathrm{p} 2$ 的确定}

上述公示中接触压强 $\mathrm{p} 1$ 和 $\mathrm{p} 2$ 是两个未知量, 可以通过 转子中各部件的配合关系来确定, 分别令 $r$ 取 $\mathrm{R}_{\mathrm{ro}}, \mathrm{R}_{\mathrm{mi}}, \mathrm{R}_{\mathrm{mo}}$, $\mathrm{R}_{\mathrm{si}}$, 得到转轴外表面、永磁体内外表面和护套内表面的径 向位移: $u_{z r o} 、 u_{m r i} 、 u_{m r o} 、 u_{s r i}$, 根据配合条件, 它们满足 如下约束:

$$
\left\{\begin{array}{l}
u_{s r i}-u_{m r o}=\delta \\
u_{m r i}-u_{z r o}=0
\end{array}\right.
$$

由式(14)即可求得 $\mathrm{p} 1$ 和 $\mathrm{p} 2$ 。

\section{3. 有限元法验证}

转子中用到材料的属性参数如表4所示, 包括两种护 套材料, 永磁体材料和转轴材料。

表4 材料参数。

\begin{tabular}{llll}
\hline & 密度 $/\left(\mathbf{k g} / \mathbf{m}^{3}\right)$ & 弹性模量 $/ \mathbf{G P a}$ & 泊松比 \\
\hline 钛合金 & 4400 & 109 & 0.34 \\
镍基合金 & 8240 & 204 & 0.3 \\
铜 & 8920 & 117.7 & 0.35 \\
永磁体 & 7550 & 175 & 0.24 \\
40CrNiMoA & 7850 & 209 & 0.295 \\
\hline
\end{tabular}

针对上文推导的转子应力解析公式, 采用有限元法进 行验证。有限元模型和解析模型中选取的转子尺寸、过盈 量、转速与材料完全一致。选取其中一组转子参数一一永 磁体内径 $40 \mathrm{~mm}$, 外径 $72.75 \mathrm{~mm}$, 护套与永磁体之间的过 盈量为 $0.1 \mathrm{~mm}$, 护套材料选取钛合金材料, 护套外径取 $87 \mathrm{~mm}$ 。通过有限元仿真得到转子中永磁体和护套的切向 应力和径向应力其结果如图2所示。

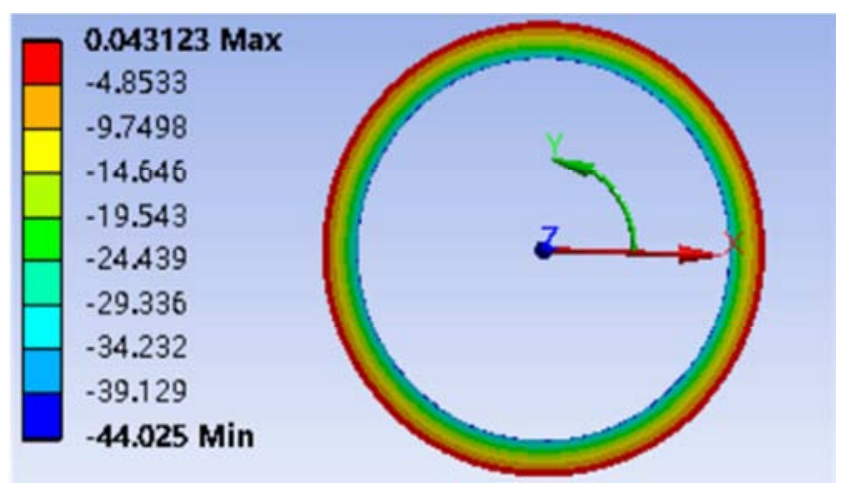

(a)护套径向应力

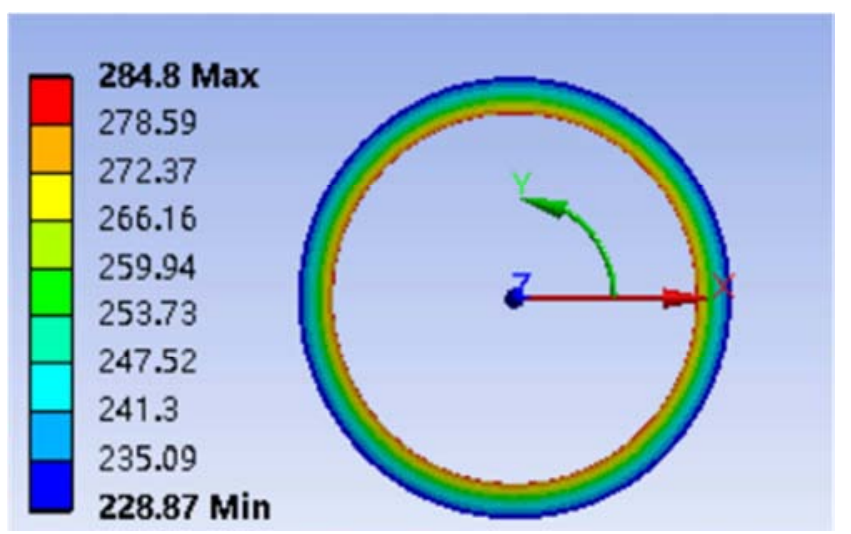

(b)护套切向应力

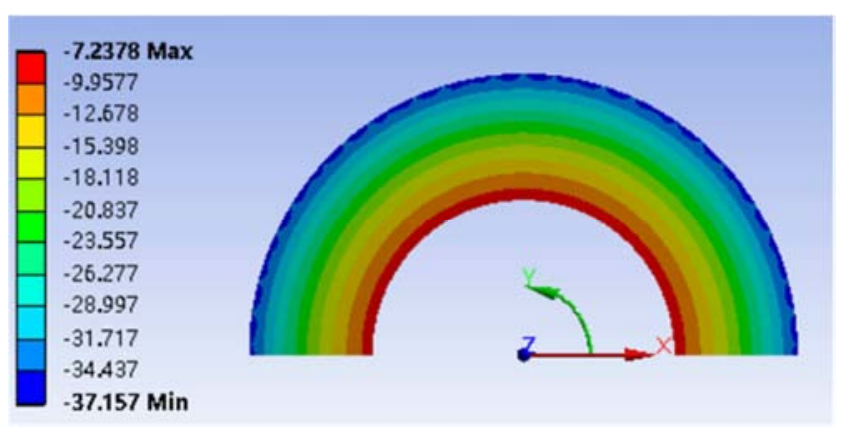

(c)永磁体径向应力

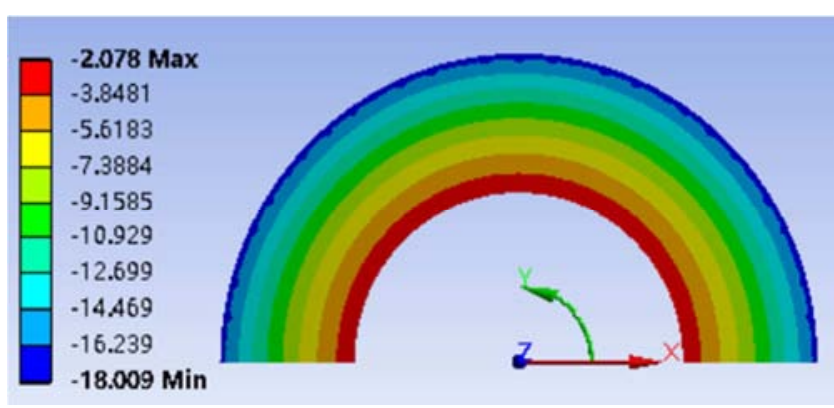

(d)永磁体切向应力

图2 转子径向和切向应力云图。

图中, $\mathrm{X}$ 方向为径向, $\mathrm{Y}$ 方向为切向, $\mathrm{Z}$ 方向为轴向。 可以看出, 护套和永磁体中的最大切向应力均产生在其各 自的内表面; 护套和永磁体的最大径向应力(绝对值)产生 在两者的结合面。

为校验解析模型的准确性, 将理论计算出的应力数据 与有限元仿真出的数据进行量化比较。沿有限元模型圆周 
方向取多组数据的平均值, 得到转子中不同半径处的径向 应力和切向应力, 与解析模型的计算值相对比, 如图 3 所

示。其中实线为理论计算数据, 散点为有限元仿真数据。

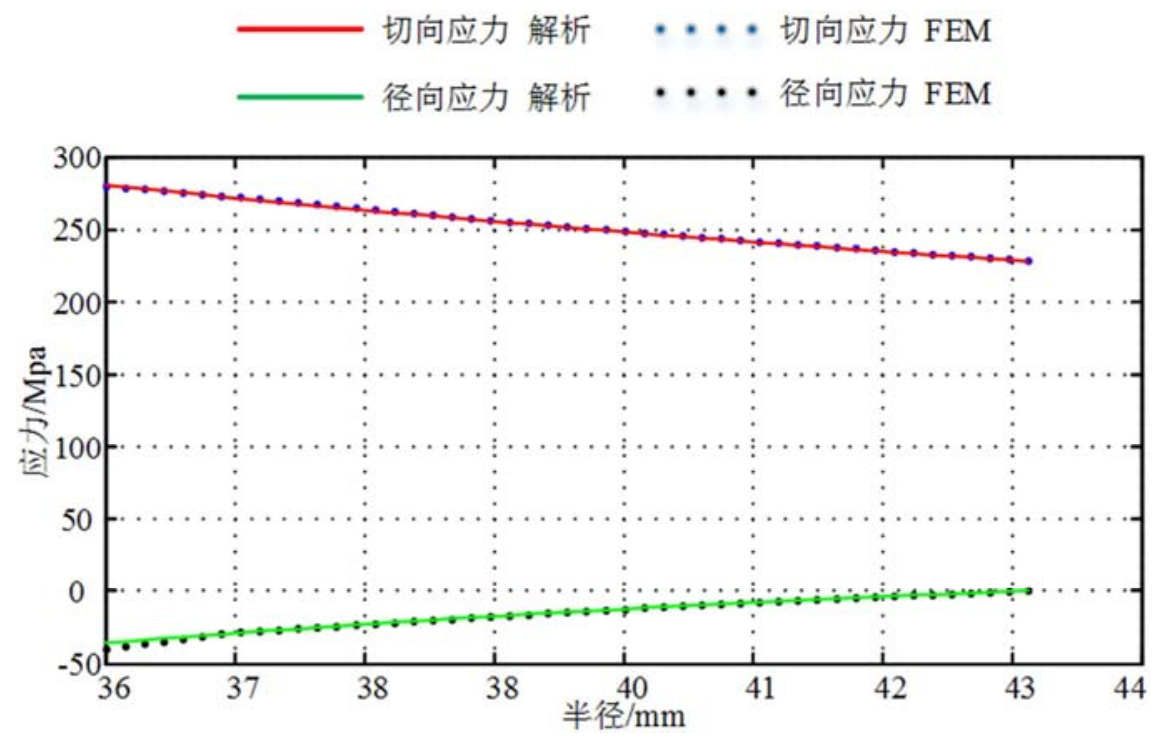

(a)护套应力分布

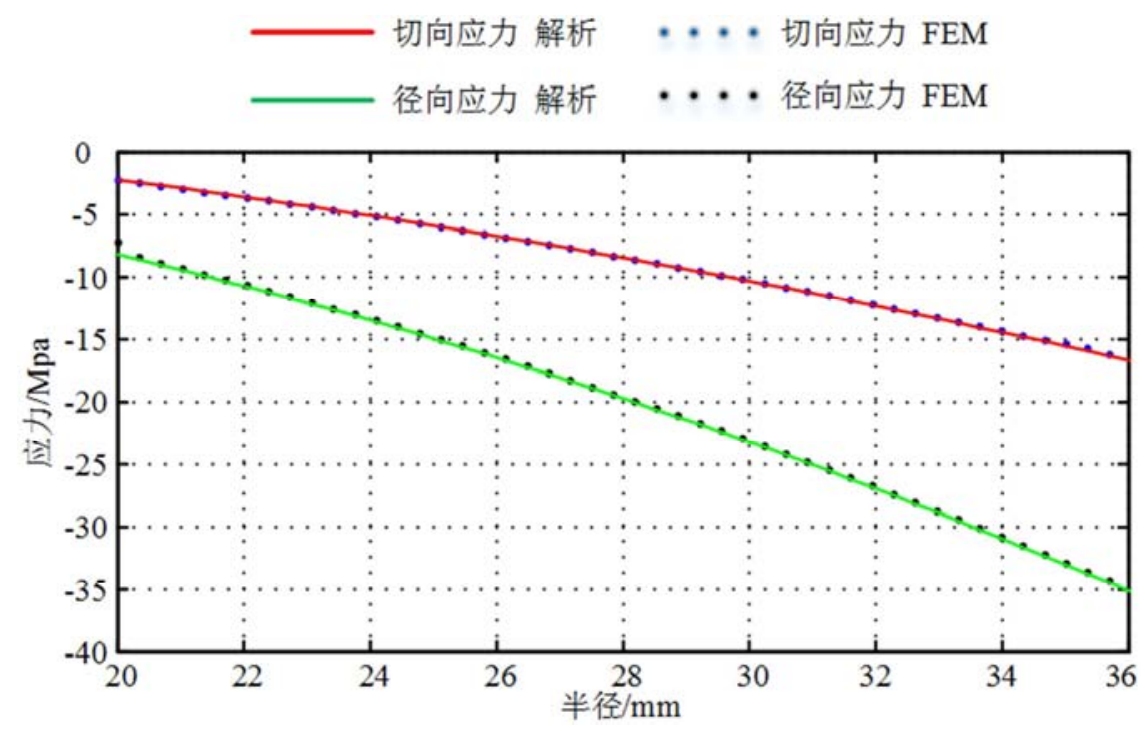

(b)永磁体应力分布

图3 理论计算与有限元法计算对比。

由上图可以看出, 理论计算和有限元仿真的数据基本 吻合, 验证了理论解析模型的准确性; 护套和永磁体中的 切向应力沿着半径方向逐渐减小; 护套中的径向应力沿半 径方向从负值逐渐增大, 至护套表面时, 径向应力为 0 ; 永磁体中的径向应力沿半径方向逐渐减小; 护套和永磁体 的应力情况均满足其安全需求, 该尺寸转子的强度设计满 足安全需求。

\section{4. 永磁体内径对转子强度的影响}

上述理论计算和有限元仿真的对比结果, 验证了解 析模型的准确性。下面基于解析模型, 分析永磁体内径
对转子应力的影响以及两种合金护套对永磁体的保护效 果。由前一节的研究, 得到了一系列永磁体尺寸, 但护 套的厚度尚未确定。这里以护套所受最大等效应力相同 为准则, 确定各永磁体尺寸下对应的护套厚度。分别采 用钛合金护套和镍基合金护套，护套与永磁体间的过盈 量取 $0.1 \mathrm{~mm}$, 由于两种护套的材料特性不同, 因此选取 的最大等效应力值也不同, 其中钛合金护套取 $285 \mathrm{MPa}$, 镍基合金护套取 $532 \mathrm{MPa}$ 。由解析模型可知，保持护套所 受最大等效应力相同, 计算出相应永磁体尺寸下对应的 护套厚度, 如图4所示。 


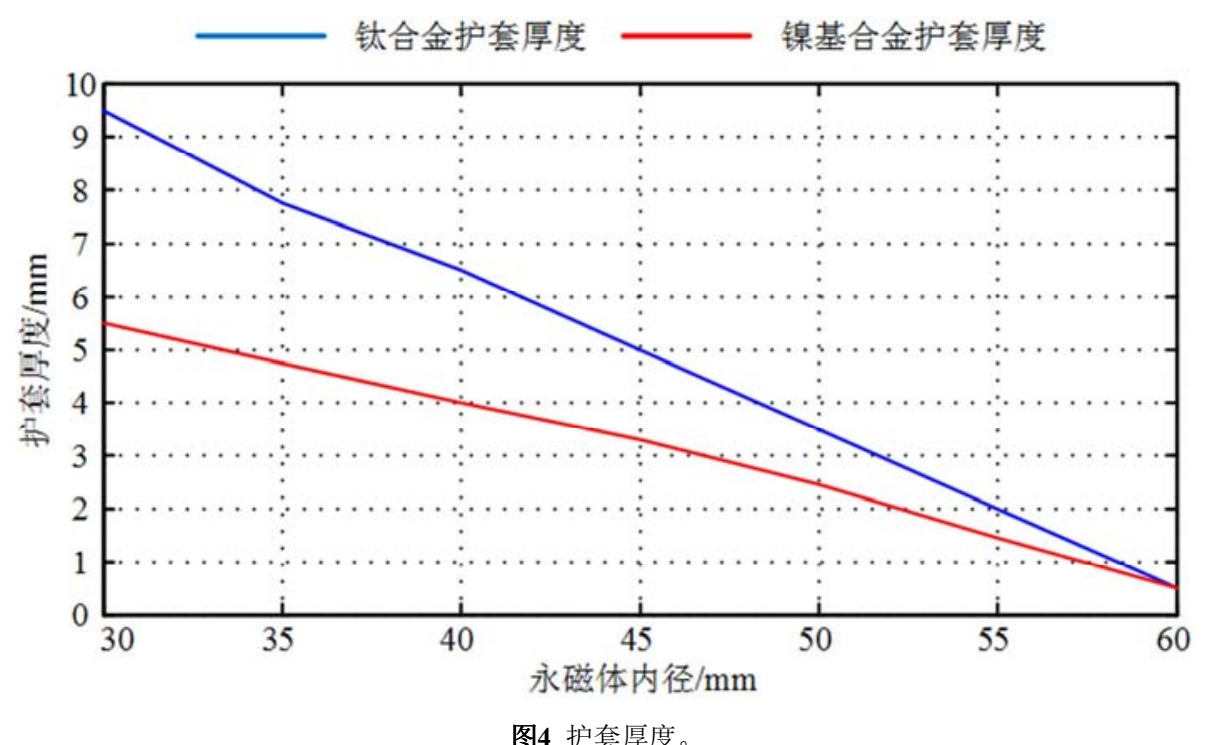

由图4可以看出, 在保护套受到的最大等效应力相同时, 随着永磁体内径的增大, 护套的厚度在减小。根据以上分 析计算数据, 可以得到一系列转子尺寸, 由这些转子尺寸, 再来分析永磁体内径对转子强度的影响, 计算出相应尺寸 下, 永磁体的最大应力, 如图5所示。

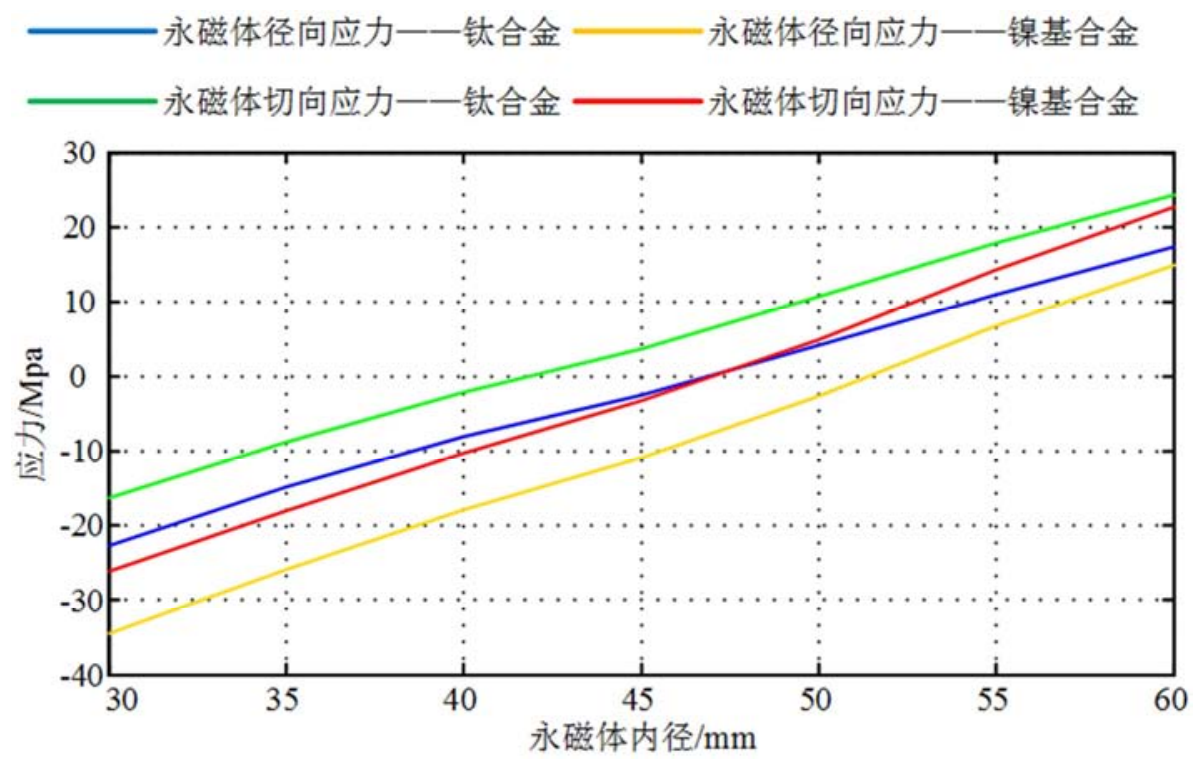

图5 永磁体应力随永磁体内径的变化。

从上述结果可以看出, 随着永磁体内径的增大, 永磁体 中的最大径向应力和最大切向应力都在增大, 转子应力恶化; 对比两种材料的护套, 在相同的永磁体尺寸和过盈量下, 镍 基合金护套转子中永磁体的受力低于钛合金护套转子中永磁 体的受力, 并且镍基合金护套的厚度低于钛合金护套的厚度, 因此镍基合金护套对永磁体的保护效果要优于钛合金护套。

\section{4. 临界转速分析}

除满足强度要求之外, 高速永磁电机转子还需具备优 良的动态性能, 必须对转子进行动力学分析, 准确计算其 临界转速。
本文采用有限元法对转子进行动力学分析。剖分单 元取 $5 \mathrm{~mm}$ 大小, 接触面的接触刚度因子取 0.05 , 并且将 转子两端的浴轮盘也考虑在内。当永磁体内径为 $40 \mathrm{~mm}$ 时, 采用两种合金护套的一阶弯曲频率仿真结果如图6 所示。改变永磁体内径, 根据上文中得到的相应的转子 尺寸, 同时采用两种护套, 对这些转子继续进行动力学 分析, 得到不同永磁体内径下, 各转子的一阶弯曲频率, 如图7所示。 


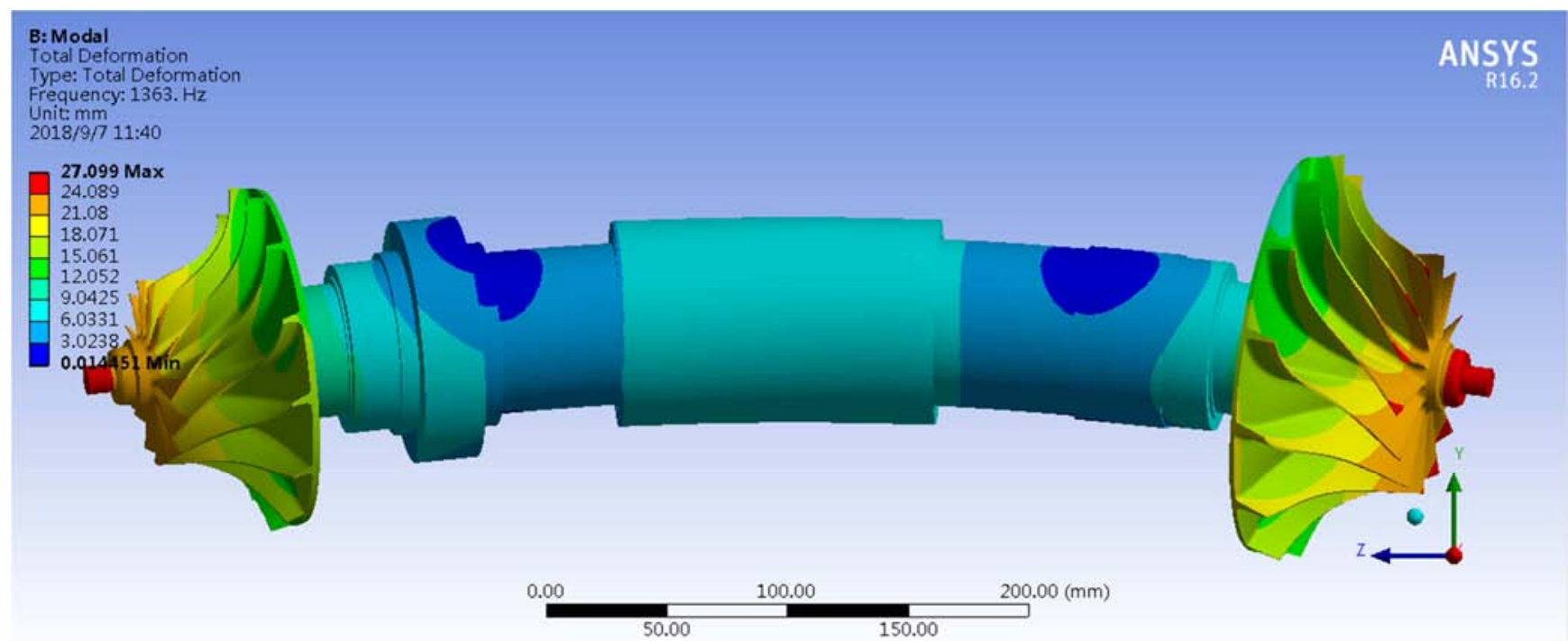

(a)钛合金护套转子一阶弯曲频率

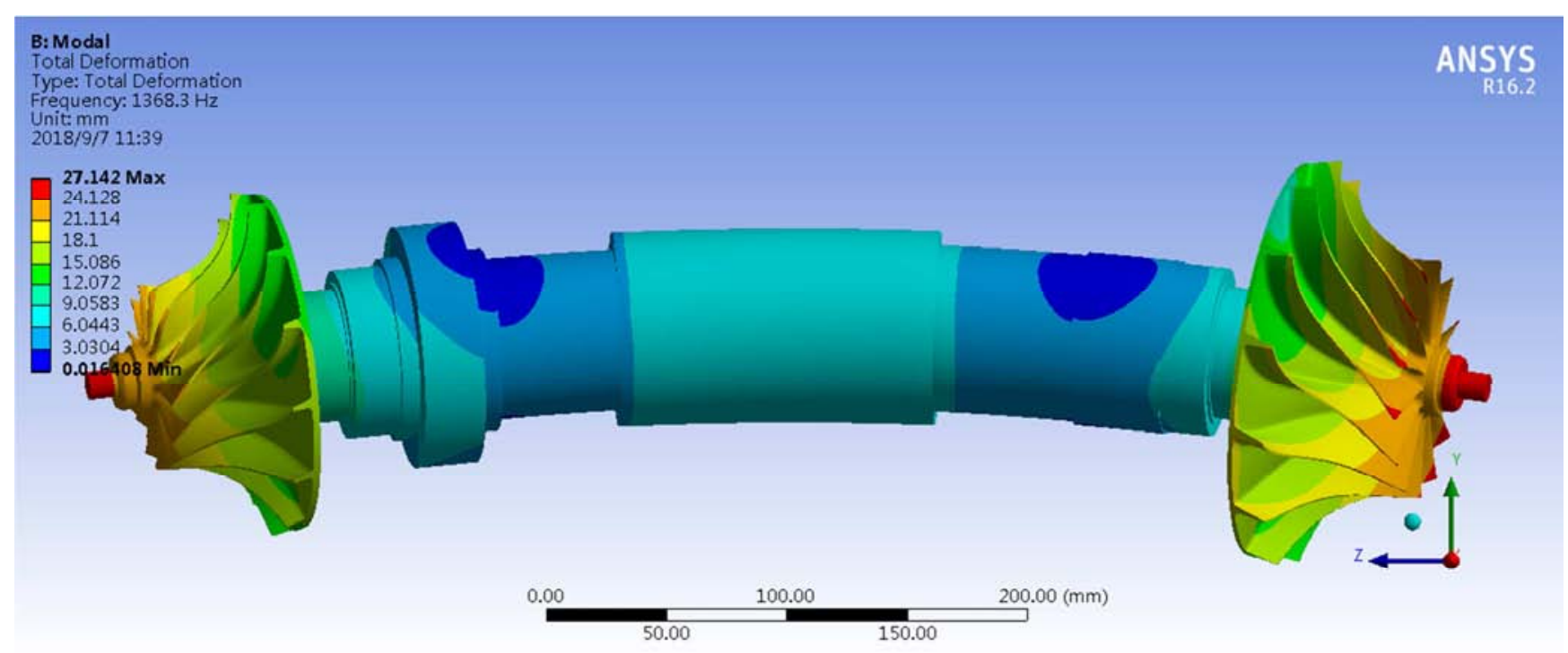

(b)镍基金护套转子一阶弯曲频率

图6 转子一阶弯曲频率(永磁体内径 $40 \mathrm{~mm}$ )。

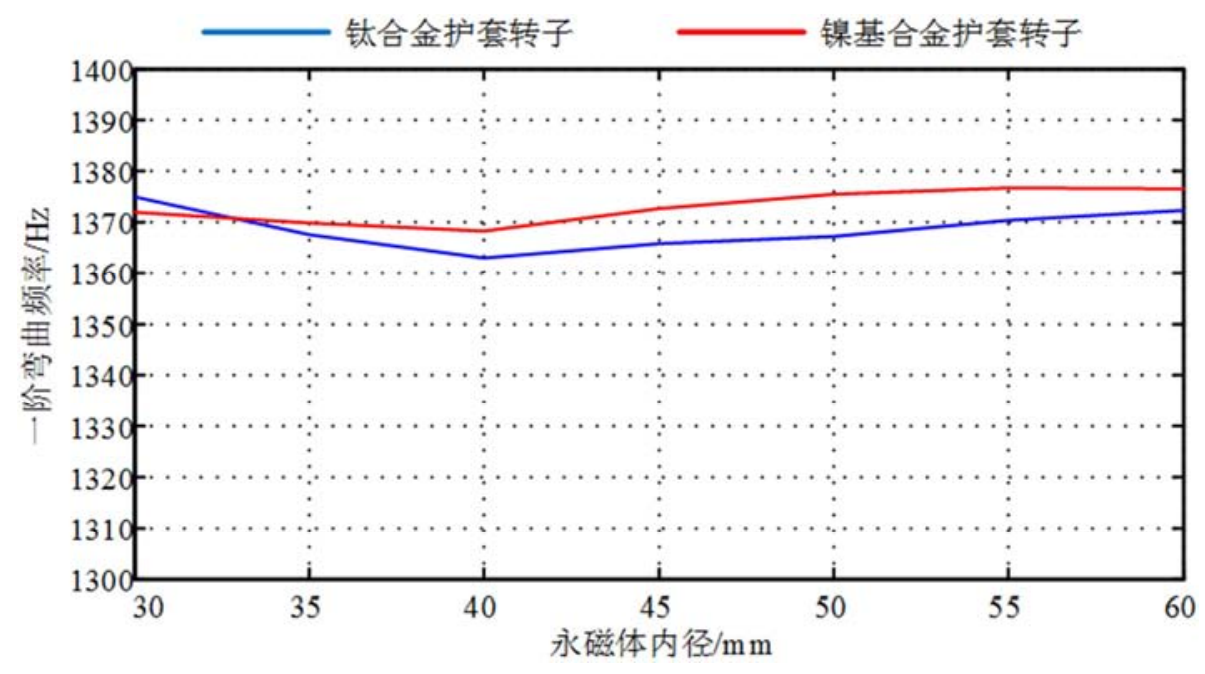

图7一阶弯曲频率。 
由以上结果可以看出, 这些转子的临界转速均满足设 计要求; 随着永磁体内径的增大, 转子的临界转速变化很 小，转子的动态性能基本不变; 采用钛合金护套和镍基合 金护套时，转子的临界转速相差很小，但镍基合金护套的 厚度更小，耗材较少。

\section{5. 结论}

本文在相同的电磁性能基础上, 从转子强度和临界转 速两方面对一台 $150 \mathrm{~kW}, 30000 \mathrm{rpm}$ 的高速永磁电机进行 了综合分析, 通过改变永磁体内径和护套材料, 对多种设 计方案进行了分析比较, 得出以下结论:

1)随着永磁体内径的增加, 永磁体所受切向应力和径 向应力都在增大, 转子应力恶化;

2)随着永磁体内径的增加, 转子的一阶临界转速变化 很小，转子的动态性能基本不变;

3)从转子应力和临界转速方面看, 采用镍基合金护套 的厚度更薄，节约材料。

\section{参考文献}

[1] 孙权贵,邓智泉,张忠明.基于齿槽效应的高速永磁电机转子 浴流损耗解析计算 [J].电工技术学报, 2018, 33(9):1994-2004。

[2] 崔刿楷,程文杰, 肖玲, 等. $10 \mathrm{~kW}$ 超高速永磁电机三维瞬态温 度场计算 $[J]$. 电机与控制应用, 2018(2)。

[3] 卢南方,张宇,杨立, 等.高速永磁无刷电机转子模态分析与试 验研究 $[J]$. 机械设计与制造, 2018(6)。

[4] 高永军,王雷.永磁同步电机控制技术研究及应用[J].铁道机 车与动车, 2018(7)。

[5] 刘威,陈进华,崔志琴, 等.高速永磁电机碳纤维护套转子综合 特性研究 [J]. 微特电机,2017(12):1-4。

[6] 董剑宁,黄允凯,金龙,等.高速永磁电机设计与分析技术综述 [J].中国电机工程学报, 2014, 34(27):4640-4653。

[7] 丁鸿昌,肖林京,张华宇,等.高速永磁电机转子护套过盈配合 量计算及应力分析 [J]. 机械设计与研究, 2011, 27(5):95-98。
[8] 张涛, 朱煜秋,孙晓东,等.基于有限元法的高速永磁转子强 度分析 [J].电机与控制学报, 2012, 16(6):63-68。

[9] Binder A, Schneider T, Klohr M. Fixation of buried and surface-mounted magnets in high-speed permanent-magnet synchronous machines[J]. IEEE Transactions on Industry Applications, 2006, 42(4):1031-1037.

[10] 邓旺群.航空发动机柔性转子动力特性及高速动平衡试验 研究[D].南京航空航天大学, 2006。

[11] 钱伟长.电机设计强度计算的理论基础 $[\mathrm{M}]$. 安徽科学技术 出版社, 1992。

[12] Aglen O, Andersson A. Thermal analysis of a high-speed generator[C]// Industry Applications Conference, 2003. Ias Meeting. Conference Record of the. IEEE, 2003:547-554 vol.1.

[13] Weeber K R, Shah M R, Sivasubramaniam K, et al. Advanced permanent magnet machines for a wide range of industrial applications[C]// Power and Energy Society General Meeting. IEEE, 2010

[14] 程文杰,耿海鹏,冯圣,等.高速永磁同步电机转子强度分析 [J] 中国电机工程学报, 2012, 32(27):87-94。

[15] 张凤阁,杜光辉,王天显,等.高速永磁电机转子不同保护措施 的强度分析 [J].中国电机工程学报, 2013, 33(z1):195-202。

[16] 张超,朱建国,韩雪岩.高速表贴式永磁电机转子强度分析 [J]. 中国电机工程学报, 2016, 36(17):4719-4727。

[17] 胡家奇. 采用碳纤维绑扎的磁悬浮高速永磁电机转子研究 [D]. 南京航空航天大学, 2013。

[18] 田拥胜,孙岩桦, 虞烈. 高速永磁电机电磁轴承转子系统的动 力学及实验研究 [J]. 中国电机工程学报, 2012, 32(9):116-123。

[19] 王继强, 王凤翔, 宗鸣. 高速电机磁力轴承-转子系统临界转速 的计算[J]. 中国电机工程学报, 2007, 27(27):94-98。

[20] 黄梓嫄,韩邦成,周银锋. 非线性接触下磁悬浮电机柔性转子 系统模态分析 [J]. 中国电机工程学报,2014, 34(15):2438-2444。 